CORRIGENDUM

doi:10.1038/nature09991

\title{
A map of human genome variation from population-scale sequencing
}

The 1000 Genomes Project Consortium

Nature 467, 1061-1073 (2010)

In this Article, Yali Xue, of the Wellcome Trust Sanger Institute, Wellcome Trust Genome Campus, Cambridge CB10 1SA, UK (Analysis group), and Reed A. Cartwright, of the Department of Ecology and Evolutionary Biology, Rice University, Houston, Texas 77251, USA (Analysis group: University of Montreal), were inadvertently omitted from the participant list. Also, the participants David Altshuler, Jonathan Keebler, Paula Kokko-Gonzales and Deborah A. Nickerson were listed incorrectly. In addition, Seungtai C. Yoon should be associated with affiliation 40 (Seaver Autism Center and Department of Psychiatry, Mount Sinai School of Medicine, New York, New York 10029, USA) and not with affiliation 42 (Department of Genetics and Genomic Sciences, Mount Sinai School of Medicine). These have been corrected in the HTML and PDF versions of the manuscript. Supplementary Information section 7.7 has also been corrected. 